\title{
Digital Signal Processing System Research and Design Based on FPGA
}

\author{
Xin Zhang \\ Jilin Business and Technology College, 130062, Changchun, China \\ zhangxinyr@126.com
}

Keywords: FPGA; DSP; Signal processing; CPLD; FLASH.

Abstract. Hardware platform of digital signal processing systems based on FPGA is researched and designed in this paper. Processing and data communication with an external SRIO is the main responsible by the DSP, and FPGA is responsible for modulation and demodulation, encoding and decoding, real-time signal processing and access control, and other functions, combination of the two complementary advantages, give full play to the high-performance DSP and FPGA flexibility. Meanwhile, the hardware platform to provide external SRIO interface and LVDS interfaces for fast data exchange system. CPLD and FPGA configuration data using a combination of analog FLASH parallel configuration mode, online loading function can be achieve by DSP.

\section{Introduction}

Digital signal processing is widely used in various fields of science and technology in today's digital signal processing, increasingly important role. With the rapid development of information technology, LSI and computers, digital signal processing technology has made rapid development. Especially dedicated digital signal processor (DSP) and FPGA development of large-scale programmable devices is to promote the rapid development of digital signal processing system design [1].

\section{FPGA Introduction and System Architecture}

FPGA Introduction. FPGA (Field-Programmable Gate Array), which is based on the PAL, GAL, CPLD and other programmable devices on the further development of the product. It is as ASIC (ASIC) in the field of a semi-custom circuits occur, which addresses the lack of custom circuits, but also overcome the limited number of the original gates of programmable devices shortcomings [2].

FPGA Selection. According to the design requirements for FPGA, dual FPGA to complete the modulation and demodulation, encoding and decoding, real-time signal processing and access control, and other functions, By comparing the functional requirements and number IO number, FPGA using Xilinx Virtex-5 Series next-generation high-end chips, choose model: XC5VSX95T-1FF1136C, XC5VSX95T has a logic module $160 \times 54$, the maximum RAM module $1.120 \mathrm{~Kb}$, DSP48E 64, CMT clock management 6 months, Rocket IO GTP 16, total IO bank 20, the largest number of IO using 680. System Architecture. Digital signal processing system framework design is shown in Figure 1:

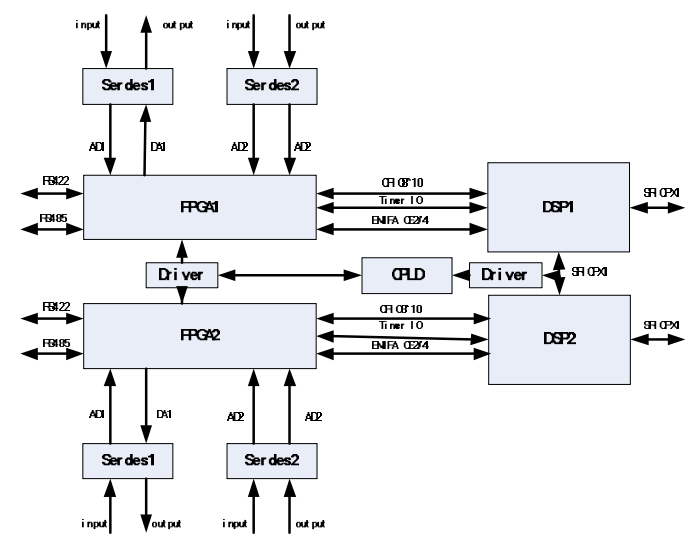

Fig1. System Architecture 


\section{Digital Signal Processing System Hardware Design}

Power Design. External 28V DC power supply used in this design, all the chip power provided by the external level shifter[3], Processing board inside the main chip operating voltage as shown in Table 1.

Table 1 .The main chip operating voltage

\begin{tabular}{llll}
\hline Device Name & Model & Quantity & \multicolumn{1}{c}{ Operating Voltage } \\
\hline DSP & TMS320C6455 & 2 & $3.3 \mathrm{~V}, 1.2 \mathrm{~V}$ \\
FPGA & XC5VSX95T & 2 & $3.3 \mathrm{~V}, 2.5 \mathrm{~V}, 1.8 \mathrm{~V}, 1.0 \mathrm{~V}$ \\
Clock Module & AD9522-2 & 1 & $3.3 \mathrm{~V}$ \\
CPLD & EPM2210 & 1 & $1.8 \mathrm{~V}, 3.3 \mathrm{~V}$ \\
SERDES & DS92LV16 & 4 & $3.3 \mathrm{~V}$ \\
FLASH & S29GL512N & 3 & $3.3 \mathrm{~V}$ \\
DDR2 & MT47H32M16BT & 4 & $1.8 \mathrm{~V}$ \\
\hline
\end{tabular}

Power conversion circuit diagram is shown in Figure 2:

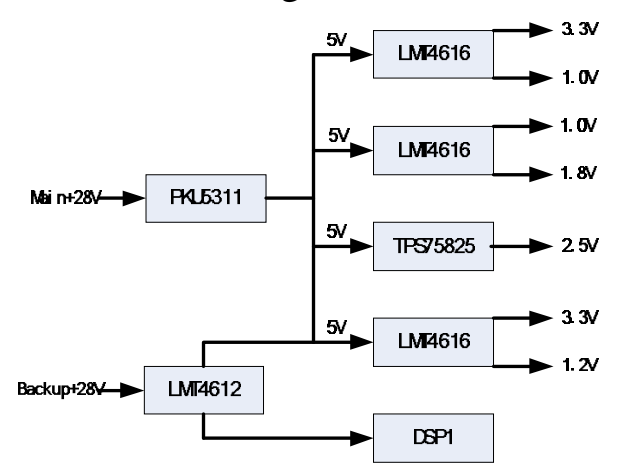

Fig2. Power Module Design

Clock Design. In a DSP + FPGA digital signal processing systems, the clock circuit is the basis of information processing, but also the main generation source of electromagnetic radiation, Its performance has a significant impact on the system can work properly, so the entire clock design is also a key part of the design process [4]. The system clock management design diagram is shown in Figure 3.

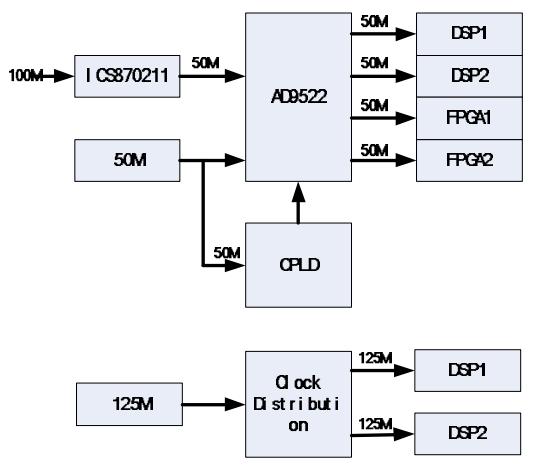

Fig3. Clock management design

CPLD Design. CPLD mainly control the reset signal, the reset FPGA and DSP, provide a manual reset function; and DSP, FPGA program loading configuration management and clock synthesizer, the first after loading FPGA according to the order to load DSP1, DSP2. It is shown in Figure 4.

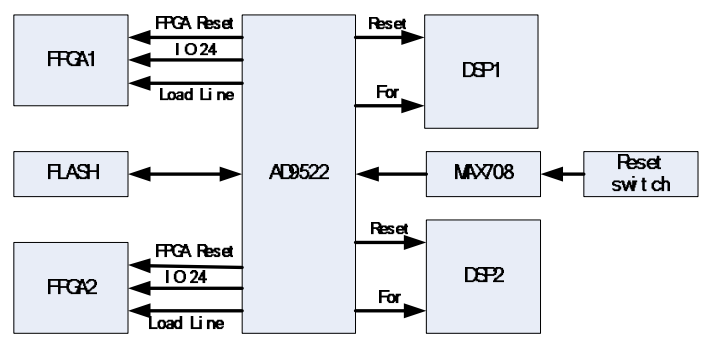

Fig4. CPLD connection diagram 
DSP Design. TI's TMS320C6455 is a high-performance 6000 series class, fixed-point digital signal processors, DSP's EMIF bus is connected to the FPGA, sharing resources, expand peripheral interface. Its connection diagram is shown in Figure 5.

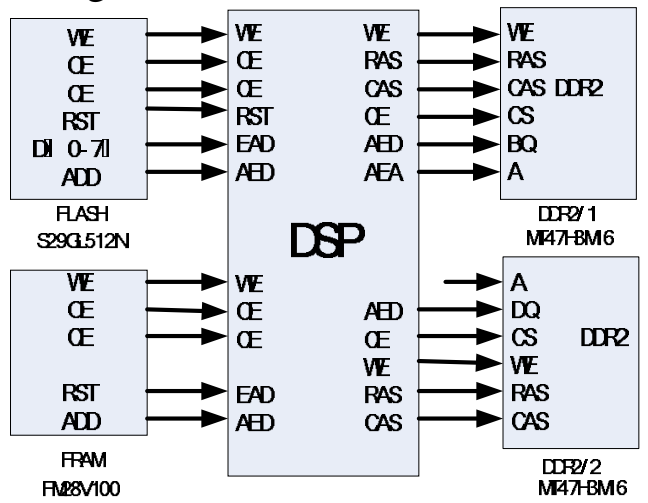

Fig5. DSP peripheral connection diagram

DSP and FPGA Interface Design. DSP chip and FPGA chip to be co-operating with the completion of several interface communication: LVDS, Communication RS422, RS485 communication, connection diagram is shown in Figure 6 [5].

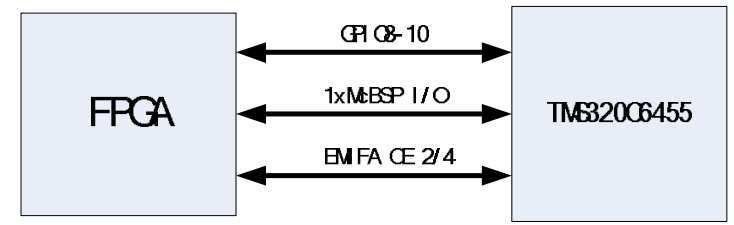

Fig6. TMS320C6455 and FPGA connections

\section{The Core Design of Digital Signal Processing Systems}

FPGA design as a whole, the core module, plays great significance in the design of the system, which greatly enhances the flexibility of the system, In this design, FPGA connected to CE2and CE4 space of DSP EMIFA bus, the TMS320C6455 full advantage of the processing power, you need to EMIFA data lines, address lines, control lines are connected with the FPGA; Under LVDS plug onto FPGA, DSP's EMIF bus to reduce stressful situations while increasing the flexibility of LVDS processing; Two FPGA needs to be loaded through the CPLD, designed with the main parallel loading mode, CPLD simulate the role of Platform FLASH, so the parallel configuration of FPGA and CPLD pin connections. Also, each FPGA external RS422 interface all the way, all the way RS485 interface and one RS232 interface [6].

LVDS Connector Interface of FPGA Design. Primarily through the high-speed LVDS circuit chip DS92LV16 to achieve their respective functions. Circuit diagram is shown in Figure 7.

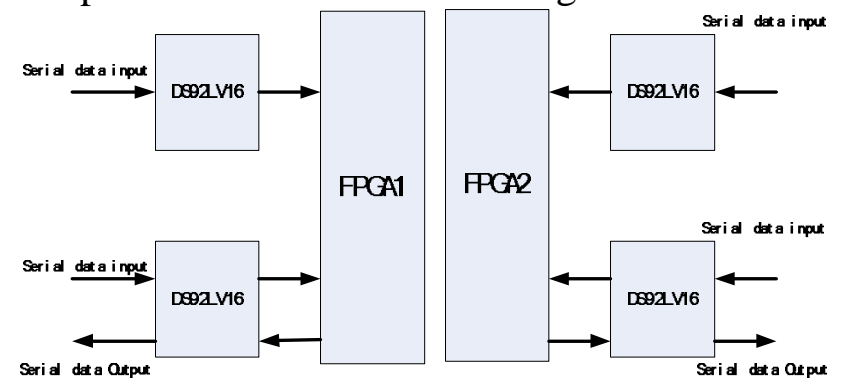

Fig7. LVDS circuit design

FPGA Design of UART Communication Interface. The design calls using FPGA IP core way to achieve UART communication, the choice in the design MAX3232ESE as RS232 signal driver / receiver, Selection MAX3491 as RS422, RS485 converter. The design uses a block diagram using the UART hardware connection as in Figure 8. 


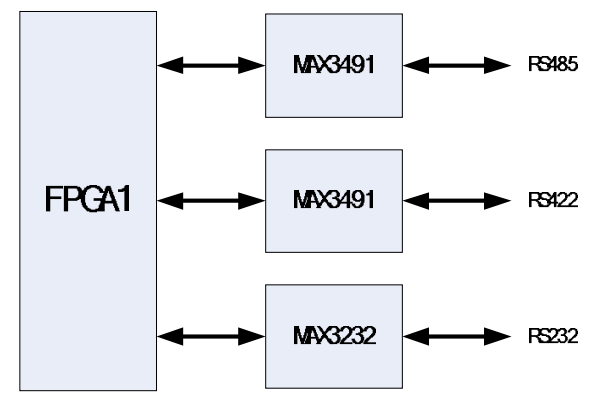

Fig8. UART communications hardware diagram

FPGA Configuration Design Data.Realization diagram shown in Figure 9, the FPGA configuration needs to load all the data lines, address lines, control lines connected to the CPLD, CPLD in the power stage, the role of simulation in parallel to load the configuration of the chip, read configuration data from FLASH, according to the timing requirements of the corresponding data to the FPGA and control signals the FPGA configuration.

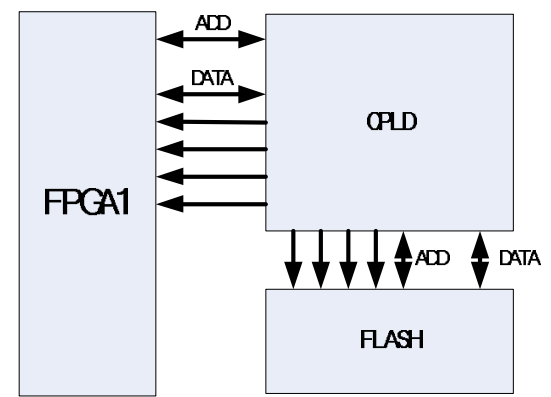

Fig9. FPGA loaded realization diagram

\section{Summary}

The rapid development of digital signal is processing in more and more application fields. DSP and FPGA development, for the development of digital signal processing plays an important role. Digital signal processing based on DSP + FPGA design is the high-performance DSP and FPGA flexibility combined with a processing capability enables the system to develop flexible. In the design of the system and the commissioning process, there are many shortcomings, some design needs further improvement.

\section{References}

[1] Y.M.Ding, X.Q.Gao. Digital Signal Processing, Xi' an University of Electronic Science and Technology Press, 2000.

[2] Zh.C.Cao, Y.T.Zhao. High-speed General-purpose Real-time Signal Processing Platform based on DSP + FPGA ,Electrical \& Electronic Education, 2010.

[3] J.L.Guo. The Design and Implementation of Signal Acquisition and Processing System based on DSP ,Wuhan University of Technology, 2009.

[4] Z.M.Fan. DDR2SDRAM Controller Design and Verification, Xi'an University of Electronic Science and Technology, 2009.

[5] G.Yang. FPGA Application and Research in Digital Signal Processing, Hunan University, 2004.

[6] Zh.Shu. DDR2 controller IP Design and FPGA Implementation, Hefei University of Technology, 2009. 